\title{
CREATIVIDAD Y ALFABETIZACIÓN INFORMACIONAL. EL DESAFÍO EN CUATRO PROPUESTAS
}

\section{Creativity and Information Literacy. The Challenge in Four Proposals}

\author{
Criatividade e alfabetização informacional. $\mathrm{O}$ desafio em quatro propostas
}

\section{RECIBIDO:25 DE OCTUBRE DE 2014}

Romina Cecilia Elisondo (Argentina)

Doctora en Psicología. Magíster en

Educación

Universidad Nacional de Río Cuarto

relisondo@gmail.com

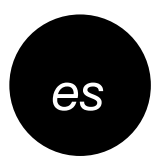

\section{RESUMEN}

Se presenta una investigación de propuestas iniciales de alfabetización informacional en cuatro grupos distintos de estudiantes de la Universidad Nacional de Río Cuarto (Argentina). Se llevaron a cabo actividades de gestión de información científica disponible en bibliotecas y en buscadores en línea especializados. Se desarrollaron encuentros presenciales e intercambios virtuales vía Facebook y aulas virtuales. Los grupos eran diversos en cuanto a edades, carreras y etapas de formación; sin embargo, no se han observado diferencias importantes respecto de conocimientos previos y procesos desarrollados durante la resolución de las actividades. Todos los grupos mostraron interés en las propuestas y destacaron la necesidad de actividades sistemáticas de gestión de información en las carreras de grado.

ACEPTADO:10 DE DICIEMBRE DE 2014

Danilo Silvio Donolo (Argentina) Doctor en Ciencias de la Educación Universidad Nacional de Río Cuarto donolo@gmail.com
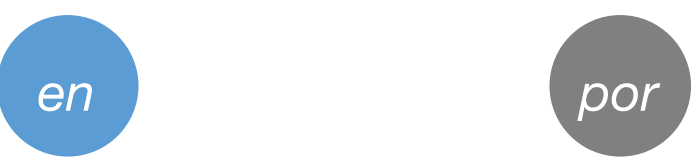

\section{ABSTRACT}

This article presents a research with some original proposals on information literacy in four different groups of students of National University of Río Cuarto (Argentina). We carried out scientific information management activities available in libraries and specialized search engines. We also developed in-person and on-line meetings -via Facebook and virtual classrooms-. The groups were diverse regarding age, major, and level of education. However, there are not significant differences with respect to previous knowledge and processes developed during the execution of the activities. The groups were interested in the proposals and highlighted the need for management systematic activities within the majors.
PALABRAS CLAVE: enseñanza alternativa, alfabetización informacional, innovación, conocimientos instrumentales, entornos virtuales.
KEYWORDS: alternative teaching, information literacy, innovation, instrumental knowledge, virtual environments.

\section{RESUMO}

Apresenta-se uma pesquisa de propostas iniciais de alfabetização informacional em quatro grupos diferentes de estudantes da Universidade Nacional de Rio Cuarto (Argentina). Levaram-se a cabo atividades de gestão de informação científica disponível em bibliotecas e em buscadores on line especializados. Desenvolveram-se encontros presenciais e intercâmbios virtuais via Facebook e aulas virtuais. Os grupos eram diversos em quanto a idades, cursos superiores e etapas de formação; porém, não se têm observado diferenças importantes respeito de conhecimentos prévios e processos desenvolvidos durante a resolução das atividades. Todos os grupos mostraram interesse nas propostas e destacaram a necessidade de atividades sistemáticas de gestão de informação nos cursos superiores de formação.

\section{PARA CITAR ESTE ARTÍCULO/TO CITE THIS ARTICLE/PARA CITAR ESTE ARTIGO:}

Elisondo, R. C. y Donolo, D. S. (2014). Creatividad y alfabetización informacional. El desafío en cuatro propuestas. Panorama, 8(15), 23-33. 


\section{INTRODUCCIÓN: CONTEXTOS, ALFABETIZACIONES $Y$ PROPUESTAS}

La vida de gran parte de la población mundial parece estar en transformación debido a las interacciones tecnológicas y los nuevos procesos comunicativos que propician. Los entornos educativos, como parte de contextos más amplios, no están ajenos a los impactos de las tecnologías en la vida cotidiana. Sin embargo, en ocasiones pareciera que las amplias potencialidades de los artefactos tecnológicos aún no son aprovechadas y resignificadas en los procesos educativos formales.

Las tecnologías y las múltiples informaciones que en ellas circulan amplían de una manera impredecible las posibilidades de aprender de las personas (Chiecher, Donolo y Córica, 2013). Asumiendo perspectivas socioculturales de la educación, entendemos que las interacciones con artefactos tecnológicos generan entornos educativos propicios para la construcción de nuevos conocimientos, el intercambio con otras personas y el desarrollo de la creatividad (Elisondo y Donolo, 2014a).

Los entornos híbridos que favorecen interacciones presenciales y virtuales configuran nuevos contextos educativos con infinitas posibilidades de comunicación y, además, suponen nuevas estructuras mentales para comprender los procesos educativos (Ballestas y Rivera, 2009). Las hipertextualidades, la confluencia de múltiples lenguajes, textos y sujetos, caracterizan los contextos actuales de comunicación (Barón, 2009). Los hipertextos constituyen ejemplos precisos de las posibilidades de aprendizaje que ofrecen las tecnologías, ya que no solo muestran textos, sino formas cognitivas alternativas alejadas de la linealidad y la simplicidad. Coincidimos con Argos y Ezquerra (2013) en la importancia de considerar la hipertextualidad como un nuevo enfoque educativo, como una nueva manera de entender los procesos educativos y los papeles e interacciones de docentes y estudiantes.

Los procesos creativos, al igual que las hipertextualidades, recorren caminos divergentes, imprevisibles y de infinitas alternativas. Los entornos híbridos caracterizados por multiplicidad de voces, textos, perspectivas y posibilidades configuran contextos promisorios para la construcción de conocimiento, pensamientos y procesos
Difícilmente un docente pueda anticipar los procedimientos, caminos y contenidos a que accederá un estudiante cuando se le solicita una búsqueda específica en internet. Por más orientaciones y recortes que se produzcan en las consignas, es imposible anticipar por dónde navegará ni qué cosas encontrará. Ilimitados, impredecibles e inesperados parecen ser los contextos educativos que se generan cuando se favorecen interacciones entre los estudiantes y las tecnologías. Esas son precisamente las características de los entornos que hemos definido como favorables para el desarrollo de la creatividad y el aprendizaje en sentido amplio (Elisondo, Donolo y Rinaudo, 2013a).

La disponibilidad de amplias cantidades de información y el dinamismo con que estas circulan en diversos medios, redes y soportes genera un nuevo contexto educativo donde crear propuestas de alfabetización informacional (Alfin) es indispensable. Enseñar y aprender a gestionar información científica y académica en diferentes entornos y con diversos fines es un propósito educativo relevante para la educación superior y la formación de grado en todas las áreas del conocimiento.

Según la Organización de las Naciones Unidas para la Educación, la Ciencia y la Cultura (Unesco, 2009), la alfabetización informacional supone capacidades de las personas y los grupos para identificar necesidades de información, localizar y evaluar su calidad, almacenar y recuperar datos y utilizar la información para crear y comunicar conocimiento. La alfabetización informacional implica la gestión eficaz y ética de la información a partir del desarrollo de competencias comunicativas, informáticas y tecnológicas.

El concepto de alfabetización digital aparece más ligado a competencias vinculadas al uso de tecnologías, y deja, a veces, de lado aspectos relativos a la comunicación y gestión de la información. Según González (2012, p. 23), "la Unesco ha optado en los últimos años por evitar el término digital y se decanta por utilizar una solución compuesta de los términos información y medios".

Desde 2008 la Unesco propone un concepto integrador: alfabetización mediática e informacional, que abarca múltiples competencias vinculadas a la comprensión, lectura crítica y producción de contenidos e informaciones en diferentes tipos de medios de comunicación y otros proveedores de información, como bibliotecas, archivos, 
museos e internet, independientemente de las tecnologías utilizadas. Asimismo, la alfabetización mediática e informacional supone una dimensión ética en el uso y la producción de contenidos e informaciones en diferentes formatos y medios (Wilson, 2012) y un importante potencial para la construcción de ciudadanías y la consolidación de democracias participativas (Culver y Jacobson, 2012; Del Moral y Villalustre, 2013; García, Ramírez y Rodríguez, 2014).

Tal como hemos explicado (Elisondo, Donolo y Rinaudo, 2013b), la alfabetización mediática e informacional se vincula con dos temáticas destacadas en la agenda de la Unesco: el libre acceso a la información científica ${ }^{1}$ y el desarrollo de recursos educativos abiertos para el aprendizaje y la enseñanza. Los recursos educativos abiertos, como los disponibles en el portal argentino $e d u c . a r$, ofrecen posibilidades para la enseñanza y el aprendizaje y también para el desarrollo de procesos creativos (Elisondo, 2013a).

La claridad y simplicidad de las definiciones de la Unesco no se corresponden con los intricados debates de los especialistas en el campo de las ciencias de la información en contextos educativos. Numerosas definiciones, algunas opuestas o contradictorias, se han construido respecto de la alfabetización informacional, inclusive algunos proponen el fin de la Alfin, o menos de los conceptos construidos al respecto (González, 2012).

Al igual que en los aprendizajes y en los procesos creativos, los límites y las fronteras entre las alfabetizaciones no son claros ni estables, se configuran de manera difusa, dinámica y conflictiva. Las únicas certezas respecto de las alfabetizaciones se vinculan con la necesidad de generar estrategias educativas acordes con el dinamismo y la complejidad con la que fluyen las informaciones en el contexto actual. Además, parece indispensable desarrollar competencias informacionales de comprensión y producción de diversos tipos de contenidos en entornos presenciales, virtuales e híbridos.

Especialistas como Area (2010), Pasadas (2010), De Pablos (2010), Area y Guarro (2012), González (2012),

1 Por eso, trabajamos para que nuestras investigaciones y producciones científicas estén disponibles y sean de acceso abierto. Dos ejemplos concretos de libros que publicamos en acceso abierto pueden apreciarse en los siguientes enlaces: http://issuu.com/ revistalatinadecomunicacion/docs/cac65 y http://issuu.com/revistalatinadecomunicacion/ docs/10cbadonolo
Moneréo y Badia (2012), Lee y So (2014) sugieren construir integraciones entre alfabetizaciones para generar comprensiones teóricas y prácticas superadoras que permitan analizar mediaciones entre sujetos y tecnologías en procesos de aprendizaje y de gestión de información diversa.

Los desarrollos teóricos actuales se refieren a la transalfabetización como convergencia de alfabetizaciones (Ipri, 2010; González, 2012), la cual diluye las fronteras entre lo digital, lo mediático, lo informacional y lo tecnológico, y supone habilidades para leer, escribir e interactuar en variadas plataformas, herramientas y medios con recursos escritos y audiovisuales. Las perspectivas de transalfabetización ponen énfasis en la participación activa de los sujetos en la producción y comunicación de informaciones, con lo cual destacan el carácter creador y social de dicha participación. Participación y creatividad en multiplicidad de contextos y lenguajes caracterizan a las transalfabetizaciones.

En este contexto de posibilidades que ofrecen las tecnologías y los nuevos entornos, entendemos que debemos ofrecer a los estudiantes herramientas conceptuales, procedimentales y éticas para gestionar la información científica y aprender en la universidad. Nuestra preocupación se ha plasmado en proyectos de investigación, transferencia y mejora de la enseñanza orientados ${ }^{2}$ a generar prácticas educativas innovadoras que promuevan la alfabetización informacional en estudiantes de grado de la Universidad Nacional de Río Cuarto.

Presentamos un estudio de propuestas iniciales de alfabetización informacional académica en contextos presenciales y virtuales con estudiantes de las carreras relacionadas con educación, psicopedagogía y prácticas docentes, quienes deberían vivenciar actividades conducentes a la alfabetización informacional, por ser ellos los responsables de integrar los futuros cuerpos docentes de instituciones educativas, ya sea como profesores y maestros, ya sea como asesores y orientadores de procesos de enseñanza. El punto no es menor, ya que da fortaleza y perspectiva prometedora al formar a formadores de formadores en propuestas e iniciativas innovadoras en

2 Proyecto Ocasiones para la creatividad y la alfabetización académica. Estudiantes, docentes, textos académicos y el juego de la escondida. Proyecto Proyectos de innovación e investigación para el mejoramiento de la enseñanza de grado, Universidad Nacional de Río Cuarto, 2013-2015. Proyecto Alfabetización informacional académica. Una propuesta para estudiantes universitarios. Programa de Transferencia de Resultados de la Investigación, Ministerio de Ciencia y Tecnología de la Provincia de Córdoba, 2014-2016. 
educación asociadas a metodologías de investigación de diseños instructivos.

\section{METODOLOGÍA: HACER E INVESTIGAR LO REALIZADO}

Elisondo I

El análisis de las propuestas educativas se realizó integrando técnicas cuantitativas y cualitativas de investigación educativa. Se analizaron y compararon las producciones de los grupos en contextos presenciales y virtuales y sus expresiones en los cuestionarios administrados durante las actividades. Interesa conocer, desde la perspectiva de los estudiantes, valoraciones, expectativas y aprendizajes vinculados a la gestión de la información académica en diferentes contextos.

En el diseño de la propuesta, recuperamos algunos avances del campo de las alfabetizaciones. Generamos actividades en contextos presenciales, virtuales e híbridos que posibilitan interacciones con compañeros y con diferentes tipos de textos científicos. Además, habilitamos espacios en redes y plataformas para propiciar intercambios entre estudiantes y también entre ellos y los docentes.

La propuesta pedagógica se sustenta en planteamientos que desatacan la importancia de la enseñanza y el aprendizaje de estrategias de búsqueda y selección de información. Específicamente, se intenta promover procesos de gestión de la información desde modelos centrados en los procesos psicológicos del buscador, es decir, donde se reconoce el papel activo de quien realizada una búsqueda (Moneréo y Fuentes, 2008). Los modelos centrados en procesos psicológicos analizan acciones, competenciales y estrategias de las personas en situaciones particulares de búsqueda. Interesan las interacciones entre los sujetos, las tecnologías y las informaciones, así como los propósitos y las situaciones en que dichas interacciones se generan.

\section{PARTICIPANTES}

Las propuestas de alfabetización informacional se desarrollaron en cuatro grupos distintos. En el segundo cuatrimestre de 2013, se trabajó con dos grupos: estudiantes de primer año de las carreras de Profesorado en Educación Inicial $(\mathrm{N}=52)$ y de quinto año de la carrera de Licenciatura en Psicopedagogía ( $\mathrm{N}=40)$. En el primer cuatrimestre de 2014, se realizaron las actividades con estudiantes de tercer año de las carreras de Licenciatura en Psicopedagogía $(\mathrm{N}=42)$ y cuarto año del Profesorado en Educación Especial $(\mathrm{N}=21)$.

\section{ACTIVIDADES}

En los cuatro grupos se desarrolló una propuesta de alfabetización informacional que incluyó cinco encuentros presenciales de dos horas y diversos encuentros virtuales vía Facebook, en grupos cerrados, y aula virtual $\mathrm{SIAT}^{3}$. Los encuentros presenciales se desarrollaron en las aulas asignadas para cada una de las materias, en las que se enmarcó la propuesta en la Biblioteca Central y en el Laboratorio de Informática de la Universidad Nacional de Río Cuarto.

En el primer encuentro presencial, se administró un cuestionario general a los estudiantes para conocer percepciones y autovaloraciones respecto de competencias vinculadas a la alfabetización informacional. Luego, los coordinadores de la propuesta presentaron diferentes formas de acceso a información académica en contextos presenciales y virtuales. En el primer encuentro también se destacó la importancia de generar estrategias para evaluar calidad de la información académica disponible en diferentes fuentes. Como cierre del primer encuentro, se presentaron algunas de las potencialidades educativas de la alfabetización informacional para la formación académica y profesional.

En el segundo encuentro se realizó una actividad de búsqueda, selección y localización de información académica en un contexto presencial: la Biblioteca Central de la Universidad Nacional de Río Cuarto. Destalles de esta actividad y de las producciones y valoraciones de los estudiantes se presentaron en un artículo publicado recientemente (Elisondo y Donolo, 2013). En la Biblioteca se les propuso a los estudiantes realizar búsquedas de diferentes tipos de textos referidos a las materias en las que se desarrolló la propuesta de alfabetización. Además de buscar los textos, los estudiantes establecieron su localización, los criterios que utilizaron para seleccionarlos y un breve comentario de cada hallazgo.

\footnotetext{
3 La plataforma SIAT ofrece espacios virtuales que dan acceso a diversos materiales, tales como textos, imágenes, videos, audio, animaciones, simulaciones, software, hipertextos, hipermedias, glosarios, etc. En las aulas virtuales, los integrantes de la asignatura pueden interactuar, proponer actividades, resolver dudas y plantear tareas de evaluación y autoevaluación. Más información en http://www.unrc.edu.ar/unrc/estudiar/aulasvirtuales.php
} 
En el tercer encuentro, se realizaron actividades de búsqueda de información en páginas web de organismos nacionales e internacionales: Unesco, portal educativo argentino educ.ar, Ministerio de Educación de la Nación Argentina, Consejo Nacional de Investigaciones Científicas y Técnicas, Universidad Nacional de Río Cuarto, etc. Los estudiantes realizaron búsquedas de informaciones referidas a proyectos educativos, recursos para la enseñanza y el aprendizaje, oportunidades de formación y trabajo, publicaciones e investigaciones en el campo educativo, entre otras búsquedas.

El cuarto encuentro presencial tuvo como objetivo principal ofrecer a los estudiantes herramientas de búsqueda de información en revistas científicas y bases de datos reconocidas en el ámbito académico, como Scielo, Latindex, Redalyc, Dialnet, Scopus, DOAJ, entre otras. Se realizaron búsquedas de artículos científicos referidos a temas específicos de las materias, en las que se desarrolla la propuesta de alfabetización. Se promovieron actividades de búsqueda, selección y recuperación de la información y de reconocimiento de diferentes estructuras y formatos de artículos de investigación. Asimismo, se establecieron diferencias entre textos de acceso abierto y documentos restringidos donde solo podían recuperar algunas partes del escrito, generalmente el resumen.

En el quinto encuentro presencial, los estudiantes realizaron búsquedas en el metabuscador de la Biblioteca Electrónica de Ciencia y Tecnología de la República Argentina. Se desarrollaron algunas estrategias específicas de búsqueda y se identificaron bases de datos disponibles y de acceso limitado a universidades nacionales y organismos científicos de Argentina. Además, respondieron a un breve cuestionario de evaluación de las actividades sobre alfabetización informacional desarrolladas en el curso.

Los encuentros presenciales se llevaron a cabo aproximadamente cada quince días. Durante todo el desarrollo de la propuesta se mantuvieron interacciones con los grupos vía Facebook y aula virtual. Los contextos virtuales fueron aprovechados por los estudiantes para plantear consultas, dudas y apreciaciones respecto de las actividades. Asimismo, los espacios virtuales permitieron a los estudiantes compartir gran parte de las informaciones y los textos encontrados.
EXPECTATIVAS INICIALES, RESULTADOS Y TESTIMONIOS

CUESTIONARIO INICLAL

Entre los resultados más importantes, observamos que todos los estudiantes destacan la importancia de disponer de competencias informacionales para su formación académica. Sin embargo, la mayoría dice tener escasas habilidades para la gestión de la información, especialmente en entornos virtuales. Entre las páginas visitadas frecuentemente por los estudiantes no aparecen bases de datos para búsquedas específicas de textos científicos o académicos. Según los estudiantes, las páginas visitadas con más frecuencia son Facebook, Twitter, YouTube, servidores de correo electrónico, Wikipedia, páginas de medios de comunicación (generalmente locales), web de la universidad para trámites administrativos (inscripción en materias, examen, etc.).

En las respuestas de los estudiantes, se observan dificultades para establecer con claridad criterios de calidad para la búsqueda, selección y organización de información académica y científica.

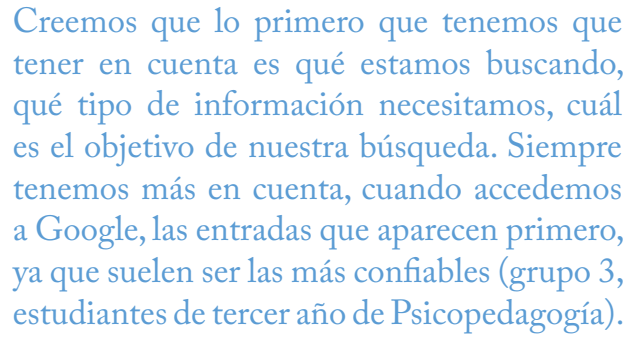

Asimismo, los estudiantes dicen tener escasos conocimientos sobre normas académicas para la escritura y la comunicación científica. Pocos comparten información sobre textos académicos y científicos en entornos virtuales. Tampoco se comparten textos y producciones grupales o individuales entre los estudiantes. Los trabajos académicos no se entienden como textos comunicativos para compartir con otros, sino solo como escritos donde el docente es el único lector y evaluador.

En los cuestionarios iniciales, los estudiantes también señalan la necesidad de propuestas educativas que promuevan la alfabetización informacional y la gestión autónoma de información científica.
Creatividad y alfabetización informacional. El desafío en cuatro propuestas 
Nos iniciamos este año en búsquedas de este tipo, cosa que nos sorprendió, porque es algo muy importante; pero, a partir de lo que sabemos sobre el uso de internet, experiencias previas con respecto a la búsqueda de información y lo visto en algunas materias de este año, consideramos que podemos buscar artículos científicos a partir de buscadores como Google Académico, bibliotecas virtuales de diferentes páginas de universidades y páginas específicas de investigación donde podemos encontrar publicaciones (grupo 5, estudiantes de quinto año de Psicopedagogía).

En el análisis del cuestionario inicial, los estudiantes también expresan tener pocas experiencias de gestión de información en contextos presenciales, como bibliotecas. La mayoría de los estudiantes, independientemente del año de cursado y la carrera, dicen haber asistido a la Biblioteca Central de la Universidad Nacional de Río Cuarto entre tres y cinco veces durante la formación académica. Las visitas a la Biblioteca generalmente tienen como propósito el estudio o la realización de algún trabajo práctico; en pocos casos se realizan búsquedas de libros o artículos científicos.

Las respuestas al cuestionario inicial demuestran la necesidad de propuestas de alfabetización informacional que promuevan en los estudiantes estrategias de búsqueda, selección, organización y producción de información científica en diferentes entornos y en la resolución de problemas diversos.

\section{PRODUCCIONES Y VALORACIONES DE LOS GRUPOS}

Los grupos realizaron interesantes búsquedas en contextos presenciales y virtuales de acceso a información científica. En la Biblioteca Central de la Universidad interactuaron con diferentes tipos de textos, autores y espacios físicos. Todos los grupos, de diferentes años y carreras, recorrieron espacios hasta el momento desconocidos de la Biblioteca, como la Hemeroteca. Antes de la actividad, ningún estudiante había buscado un artículo de revista en la Biblioteca Central. Durante la propuesta, se observó a los estudiantes interactuar con revistas, realizar lecturas generales y búsquedas específicas de textos de interés. En la visita a la Biblioteca, los estudiantes se mostraron motivados por la búsqueda de los diferentes tipos de textos solicitados y, en algunos casos, sorprendidos por los hallazgos logrados. Todos los grupos valoraron positivamente la experiencia de aprendizaje en la Biblioteca de la Universidad.

Para nosotras la actividad ha sido muy
gratificante, ya que nos sirvió para conocer
más sobre la Biblioteca, las ubicaciones de
los libros, las revistas, los diarios, y, a su vez,
nos permitió vivir una actividad diferente y
más dinámica de la que se vive siempre. Nos
sentimos con esta actividad y consideramos
que es una buena forma de incentivar a los
alumnos a recurrir más a la Biblioteca y a
sacarle provecho a este beneficio que nos
brinda la Universidad (grupo 8, estudiantes
de primer año de Educación Inicial).

En los entornos virtuales, los estudiantes también buscaron y seleccionaron distintos tipos de textos y recursos educativos. Además, reconocieron diferentes tipos de acceso a la información (abierto y restringido) y diversas bases de datos científicas. Durante la realización de las actividades virtuales, los estudiantes también se mostraron interesados por los textos a los que iban accediendo y destacaron la gran cantidad y diversidad de documentos educativos y científicos disponibles en la red. Todos los participantes afirmaron que era la primera vez que accedían a las bases de datos propuestas y también a algunas páginas con información educativa, como educ. ar. Los estudiantes se mostraron muy interesados en los recursos educativos disponibles en dicho portal y se sorprendieron por la amplitud y heterogeneidad de propuestas de enseñanza y aprendizaje desarrolladas en el sitio.

Durante las actividades, los docentes fueron orientando respecto del uso de palabras clave, el establecimiento de criterios de búsquedas avanzadas y estrategias de recuperación de información. También se trabajaron criterios más específicos de selección de la información vinculados a los contenidos y objetivos de cada una de las materias, en las cuales se desarrollaron las propuestas. Por ejemplo, en las asignaturas vinculas a la psicometría, los docentes orientaron respecto de la búsqueda de textos científicos referidos a la construcción, la validación y el uso de pruebas en diferentes grupos y contextos.

Sin embargo, se considera necesario profundizar en el establecimiento de criterios de selección de información científica y en las infinitas posibilidades de acceso a la información. En muchos casos, se observó que los estudiantes resolvían las actividades recurriendo a los 
primeros textos que aparecían en los buscadores sin profundizar en otros artículos posibles y recorrer las amplias listas de resultados de búsqueda. Quizá, para próximas propuestas, resulte relevante proponer actividades complejas de búsqueda de información que consideran varios criterios de manera simultánea e interpele a los estudiantes a desarrollar estrategias de selección más específicas. También se considera interesante desarrollar intervenciones docentes que promuevan la lectura de más cantidad de textos, no solo los primeros que aparecen, reconociendo el carácter ilimitado y dinámico de los aprendizajes mediados por tecnologías. Es decir, promover más oportunidades de aprendizaje y, por lo tanto, más ocasiones de creatividad.

Asimismo, parece relevante profundizar en las propuestas aspectos vinculados a compartir la información. Sin bien las actividades se compartían en Facebook y en los foros de la plataforma educativa virtual SIAT, no se observaron comentarios, apreciaciones ni consultas de los estudiantes respecto de las producciones de otros grupos. Solo aparecieron unos pocos "me gusta" en algunas respuestas, especialmente cuando se compartieron los recursos educativos encontrados en educ.ar. Tal vez convenga también revisar los encuadres de las actividades, ya que estas parecen ser solo para el docente, para la evaluación, y las producciones de los grupos no son visualizadas como informaciones para compartir con toda la clase. Quizá sea necesario generar estrategias de reflexión en cuanto a los planes cognitivos elaborados por los estudiantes respecto de los alcances de las tareas y los múltiples interlocutores posibles.

Compartir y comentar es una acción muy desarrollada en los contextos virtuales y en las redes sociales; sin embargo, a la hora de las actividades académicas, estas no parecen ser prácticas muy usuales entre los estudiantes. Entendemos que las propuestas de alfabetización informacional deben poner énfasis en el aspecto comunicativo y colaborativo de la gestión de la información con diversos propósitos e interlocutores, en diferentes contextos y situaciones. Algunos pocos "me gusta" parecen como procesos iniciales de comunicación entre los estudiantes, y la ausencia de comentarios y de cuestiones compartidas muestra una interesante línea de trabajo en las propuestas de alfabetización.

Los estudiantes también valoraron positivamente las propuestas de gestión de información en contextos

virtuales. Las evaluaciones ponen énfasis en la importancia de la propuesta en la formación académica y el deseo de seguir aprendiendo respecto de estrategias de búsqueda, selección, organización y producción de información. Todas las valoraciones destacan la importancia de las propuestas desarrolladas y demuestran el interés de los estudiantes por este tipo de actividad. La mayoría de los grupos expresa la necesidad de propuestas más sistemáticas e integradas a los programas y planes de estudio de la carrera. Asimismo, los estudiantes visualizan relevantes aportes de las estrategias trabajadas para la formación académica, el trabajo final de licenciatura y el futuro desempeño profesional. Los grupos también mencionan algunas dificultades en las propuestas especialmente vinculadas a la extensión y complejidad de la propuesta y la falta de tiempo para el desarrollo de las actividades. En general, las valoraciones son positivas y dan cuenta del interés de los estudiantes en las propuestas desarrolladas, independientemente del año de formación que transitan y las carreras que estudian.

\begin{abstract}
Nos gustó poder realizar esta actividad, ya que es una experiencia nueva por la que hemos transitado, puesto que a lo largo de estos años de cursado de la carrera nunca se nos invitó a realizar una actividad que consista en la búsqueda de información a través de diferentes medios. Además, nos gustó, ya que nos dio la posibilidad de conocer y pensar los diferentes medios y buscadores que se encuentran en internet acerca de textos científicos sobre nuestra disciplina y textos afines a nuestro objeto de estudio. También esta actividad nos permitió pensar sobre la calidad y seriedad de la información que vamos obteniendo de esta gran red de información que es internet, y así poder aprender nuevas estrategias en el momento de buscar información. Nos gustaría seguir aprendiendo aún más estrategias para una mejor búsqueda de información que sea confiable y válida (grupo 3, estudiantes de quinto año de Psicopedagogía).
\end{abstract}

Las producciones/valoraciones que han realizado los grupos dan cuenta de la importancia de las propuestas de alfabetización informacional en contextos universitarios, ya que los estudiantes acceden a conocimientos conceptuales, procedimentales y actitudinales necesarios para el aprendizaje, en general, y la gestión de la información en diferentes entornos, en particular. Resulta significativo que los grupos nunca hayan desarrollado actividades similares durante la formación académica,
| Creatividad

y alfabetización

informacional.

El desafío

en cuatro

propuestas 
Romina Cecilia

Elisondo I

Danilo Silvio

Donolo I

Donolot

ni siquiera los estudiantes de último año de Psicopedagogía. Pareciera que la formación académica está centrada en la enseñanza de contenidos conceptuales establecidos y no en la generación de competencias para la gestión autónoma de la información, competencias que, indudablemente, potencian el aprendizaje y ofrecen posibilidades de construir conocimientos. Los procesos de enseñanza y aprendizaje parecen aún no reconocer las posibilidades de los estudiantes de gestionar información de manera autónoma y responsable, así como de decidir acerca de los contenidos que se pueden leer, escuchar y estudiar. Que docentes y estudiantes compartan informaciones y conocimientos, posibilidades de acceso a diferentes textos y contenidos, no es una práctica muy frecuente en algunos contextos educativos. Solo la voz del docente es la autorizada para decir qué contenidos, qué formatos y en qué contextos aprender. Las tecnologías habilitan espacios para aprender y compartir información, los estudiantes tienen acceso a múltiples conocimientos, lenguajes y propuestas que podrían ser puestas en común en las clases, presenciales o virtuales, para ampliar las posibilidades de aprender de docentes y compañeros.

"Me gusta", "comentar" y "compartir” son acciones básicas de Facebook, permanentemente realizadas por los usuarios, que permiten expresar emociones e ideas y efectuar intercambios. Estas acciones son menos frecuentes en los contextos educativos, porque suelen haber pocos espacios para expresar emociones, comunicarse y compartir ideas, contenidos y posibilidades de aprendizaje.

\section{DISCUSIÓN E INTERPRETACIONES POSIBLES}

"Compartir" creemos que es una buena idea para ampliar las posibilidades de aprendizaje y creatividad en los contextos educativos. Los artefactos tecnológicos ofrecen oportunidades para que todos los participantes de los encuentros educativos puedan enseñar y aprender al mismo tiempo. Los conocimientos disponibles en las redes generan contextos educativos donde compartir es indispensable para generar innovaciones educativas y aprender en sentido amplio. Enseñar y aprender no son actividades aisladas, sino que se vinculan en todo acto educativo.

Las tecnologías ofrecen ocasiones para crear contextos 30 | comunidades de aprendizaje (Peña et al., 2011), donde todos pueden enseñar y aprender diversos contenidos, leer y escribir diferentes textos, producir y reproducir informaciones en múltiples formatos y entornos. Además, en los contextos virtuales se habilitan espacios interactivos para el desarrollo de la autonomía (Sierra, 2011). En las propuestas llevadas a cabo, los docentes promueven procesos autónomos y colaborativos en los grupos, sin dejar de orientar, ayudar y ofrecer andamios. Los docentes también propician reflexiones metacognitivas respecto de los procedimientos desarrollados y los aprendizajes construidos.

En escritos anteriores nos hemos referido a la importancia de construir ocasiones para la creatividad en contextos educativos (Elisondo, Donolo y Rinaudo, 2009) y también a las potencialidades de las tecnologías y del carácter ilimitado de los aprendizajes (Elisondo y Donolo, 2014a). Desde diferentes perspectivas y posturas, los numerosos investigadores se ocupan de las relaciones entre creatividad y tecnologías (López y Aguaded, 2015; Chibás, Borroto y Almeida, 2014; Steinbeck, 2011; Peña et al., 2011; Toro, 2004). La diversidad y complejidad de aspectos cognitivos, emocionales y comunicativos que se desarrollan en interacción con artefactos tecnológicos generan un contexto promisorio para la creatividad y la construcción de prácticas docentes alternativas y propuestas innovadoras. Según Gutiérrez y Tyner (2012), creatividad, junto con comprensión, pensamiento crítico, creatividad, conciencia intercultural y ciudadanía, son competencias básicas de la alfabetización mediática e informacional.

Las mediaciones tecnológicas habilitan entornos educativos donde se promueve la diversidad de perspectivas, de procesos de conocimiento y de creación de nuevas informaciones y productos. Es decir, las mediaciones tecnológicas pueden contribuir en la construcción de contextos creativos en enseñanza y aprendizaje donde existan ocasiones para la creatividad y para compartir con otros. Compartir es una buena idea para la creatividad, en cuanto no hay procesos creativos sin intercambios con otros, con objetos culturales y con conocimientos construidos socialmente.

Las perspectivas socioculturales de estudio de la creatividad (Glaveanu, 2010, 2014) resultan interesantes para comprender las mediaciones tecnológicas y su impacto en la educación. Tal como se sostiene desde 
estas perspectivas, los procesos creativos siempre dependen de interacciones entre sujetos, objetos culturales y conocimientos disponibles en una cultura. Las tecnologías generan un marco de interacción con otros y con producciones diversas que resultarían propicias para el desarrollo de procesos creativos.

Las propuestas de alfabetización informacional pretenden mostrar a los estudiantes posibilidades de aprender con otros, de acceder a informaciones y conocimientos construidos por diferentes comunidades científicas y académicas. Las propuestas muestran diferentes mundos, contenidos y estrategias para seguir aprendiendo. Considerando que la lectura y la disponibilidad de conocimientos variados son componentes indispensables en los procesos creativos cotidianos y de creadores reconocidos socialmente (Elisondo y Donolo, 2014b; Elisondo, 2013b), entendemos que las propuestas de gestión de la información potencian la creatividad. Asimismo, las propuestas tienen importantes componentes inesperados, es decir, son inesperadas las consignas, los resultados y los procesos desarrollados. Considerando las relaciones entre lo inesperado, lo ilimitado y la creatividad (Elisondo, Donolo y Rinaudo, 2013a; Elisondo y Donolo, 2014a), entendemos que las propuestas generan ocasiones para la creatividad.

Comunidades de aprendizaje, trabajo colaborativo, autonomía, hipertextualidad, producción de contenidos, impredecibilidad, dinamismo y complejidad son palabras que se articulan en la búsqueda de relaciones entre creatividad y alfabetizaciones o transalfabetizaciones en contextos híbridos. Las actividades desarrolladas, las expresiones y las producciones de los participantes muestran vínculos entre creatividad y alfabetizaciones en diversos contextos.

\section{CONSIDERACIONES FINALES}

Generar propuestas educativas de alfabetización informacional en contextos universitarios es una estrategia necesaria para la construcción de contextos innovadores de enseñanza y aprendizaje, donde se asigne el papel protagónico a los estudiantes y se ofrezcan múltiples posibilidades de interacciones con objetos culturales, tecnológicos e informaciones disponibles en diferentes contextos. Consideramos indispensable brindar a los estudiantes procedimientos y estrategias generales de gestión de las informaciones en el contexto actual de permanente circulación de datos, mensajes y conocimientos en diferentes formatos y redes. Desde nuestro punto de vista, es una forma de propiciar el aprendizaje permanente y la lectura crítica de los conocimientos disponibles, asimismo es ofrecer a los futuros profesionales herramientas indispensables para el ejercicio de sus prácticas de una manera informada, actualizada y que atienda a los avances en cada campo de conocimiento. Observamos numerosas potencialidades educativas en las propuestas de alfabetización informacional en cuanto propician el aprendizaje de estrategias de aprendizaje activo, autónomo y permanente, así como el uso responsable y ético de los conocimientos y de las tecnologías donde estos circulan.

Las propuestas desarrolladas amplían las posibilidades de aprender de los estudiantes ofreciendo procedimientos alternativos y originales para gestionar la información. En líneas generales, podemos afirmar que se trata de innovaciones en la enseñanza en cuanto no se observan experiencias previas de este tipo en la formación de grado de los estudiantes de las carreras señaladas. Asimismo estas propuestas generan contextos innovadores de enseñanza, trascienden los espacios áulicos y muestran diferentes formas de aprender a partir de la interacción con recursos tecnológicos y el grupo de pares. También ofrecen oportunidades de interactuar con otros y con textos científicos en diferentes formatos, fuentes y contextos de producciones y distribución. Entendemos que se trata de una propuesta inclusiva que atiende a diversas formas de aprender y genera ocasiones, también diversas, para crear nuevos pensamientos, procedimientos y conocimientos.

Los cuatro grupos eran diversos en cuanto a edades, carreras y etapas de formación; sin embargo, no se han observado diferencias importantes en cuanto a conocimientos previos y procesos desarrollados durante la resolución de las actividades. Resulta significativo que ningún estudiante haya participado de actividades similares, especialmente considerando que uno de los grupos estaba transitando la etapa final de la carrera. Todos los grupos mostraron interés en las propuestas y destacaron la necesidad de más actividades de gestión de información científica. Asimismo, pusieron énfasis en la importancia de la alfabetización informacional y en la formación académica para el desarrollo de los trabajos finales de licenciatura y también para la práctica profesional. 
Romina Cecilia

Elisondo I curriculares específicos de las materias y las propuestas
de alfabetización informacional. También es relevante lograr integraciones entre diferentes materias y hacer que las propuestas de alfabetización informacional atraviesen el currículo de la formación de grado para generar espacios interdisciplinarios, transdisciplinarios e indisciplinados para la creatividad.

Hemos compartido algunas consideraciones finales. Esperamos en nuestras casillas de correo electrónico, relisondo@gmail.com y donolo@gmail.com, sus comentarios y apreciaciones. También quisiéramos que ustedes compartan con nosotros propuestas, experiencias y desafíos para la construcción de innovaciones y transformaciones educativas.

\section{REFERENCIAS BIBLIOGRÁFICAS}

1. Area Moreira, M. (coord.) (2010). ¿Por qué formar en competencias informacionales y digitales en la educación superior? Revista de Universidad y Sociedad del Conocimiento, 7(2). Recuperado de http://rusc.uoc.edu/ojs/index. $\mathrm{php} / \mathrm{rusc} /$ article/view/v7n2-area/v7n2-area

2. Area Moreira, M. y Guarro, A. (2012). La alfabetización informacional y digital: fundamentos pedagógicos para la enseñanza y el aprendizaje competente. Revista Española de Documentación Cientifica, número monográfico, 46-74.

3. Argos, J. y Ezquerra, P. (2013). Entornos hipertextuales y educación. TESI, 14(3), 175-190.

4. Ballestas, N. y Rivera, P. (2009). La hibridación tecnológica. Revista Panorama, 7, 21-27.

5. Barón, M. (2009). Hacia una sociedad hipertextual. Revista Panorama, 7, 38-45.

6. Chibás, F., Borroto, G. y Almeida, F. (2014) Gestión de la creatividad en entornos virtuales de aprendizaje colaborativos: un proyecto corporativo de EAD. Comunicar, 43, 143-151.

7. Chiecher, A., Donolo, S.y Córica, J. (2013). Entornos virtuales de aprendizaje: nuevas perspectivas de estudio e investigaciones. Mendoza: Editorial Virtual Argentina.
8. Culver, S. y Jacobson, T. (2012). Alfabetización mediática como método para fomentar la participación cívica. Comunicar, 39, 73-80.

9. Del Moral, M. E. y Villalustre, L. (2013) Alfabetización mediática, participación y responsabilidad frente a los medios de la generación del silencio. Comunicar, 40, 173-181.

10. De Pablos, J. (2010). Universidad y sociedad del conocimiento. Las competencias informacionales y digitales. Revista de Universidad y Sociedad del Conocimiento, 7(2). Recuperado de http://www. redalyc.org/articulo.oa?id $=78016225013$

11. Elisondo, R. (2013a). ¡Hice un collage como Antonio Berni! Recursos educativos abiertos, arte y creatividad. En J. Crespo (coord.), Estudios sobre arte actual (pp. 117-144). Málaga: Eumed. Recuperado de http://dialnet.unirioja.es/servlet/ articulo?codigo $=4347027$

12. Elisondo, R. (2013b). Potencialidades creativas en contextos cotidianos. En D. Donolo y R. Elisondo (coords.), Estudio de creatividad. Las travesias de Alfonsina, de Astor, de Julios y de Marias (pp. 47-390). La Laguna, Tenerife: Sociedad Latina de Comunicación Social. Recuperado de http://www.cuadernosartesanos. org/10CBA.pdf

13. Elisondo, R. y Donolo, D. (2013). La lámpara maravillosa y la creatividad. Experiencias en la biblioteca. Creatividad y Sociedad, 21. Recuperado de http://www.creatividadysociedad. com/articulos/21/7.\%20La\%20lampara\%20 maravillosa\%20y\%20la\%20creatividad. $\% 20$ Experiencias\%20en\%20la\%20biblioteca.pdf

14. Elisondo, R. y Donolo, D. (2014a). Sin límites ni fronteras. Ideas para permitir la creatividad en educación. En P. Paoloni, M. Rinaudo, y A. González, Cuestiones en psicologia educacional. Perspectivas teóricas y metodológicas orientadas a la mejora de la práctica educativa (pp. 133-162). La Laguna, Tenerife: Sociedad Latina de Comunicación Social.

15. Elisondo, R. y Donolo, D. (2014b). Creatividad e innovación. ¿Cómo lo hacen en investigación y ciencia? La Laguna, Tenerife: Sociedad Latina de Comunicación Social. Recuperado de http:// issuu.com/revistalatinadecomunicacion/docs/ cac65

16. Elisondo, R., Donolo, D. y Rinaudo, M. (2013a). The unexpected and education: curriculums for creativity. Creative Education, 4(12B), 11-15. Recuperado de http://www.scirp.org/journal/ PaperInformation.aspx?PaperID $=41270 \#$. VNEEk8aTvJw 
17. Elisondo, R., Donolo, D. y Rinaudo, M. (2013b). No sabemos ni cómo, ni dónde buscar. Alfabetizaciones, tecnologías y ciencias. En Tercer Congreso Internacional de Comunicación Pública de la Ciencia. Rosario, Argentina: Universidad Nacional de Rosario. Recuperado de https://copuci.files.wordpress.com/2014/09/ ebook-copuci-2013_congreso-internacional-de-comunicacic3b3n-pc3bablica-de-la-ciencia_unrosario.pdf

18. Elisondo, R., Donolo, D. y Rinaudo, M. (2009). Ocasiones para la creatividad en contextos de educación superior. Revista de Docencia Universitaria, 4. Recuperado de http://www. um.es/ead/Red_U/4/elisondo.pdf

19. García, R., Ramírez, A. y Rodríguez, M. (2014). Educación en alfabetización mediática para una nueva ciudadanía prosumidora. Comunicar, 43, 15-23.

20. Glaveanu, V. (2010). Paradigms in the study of creativity: introducing the perspective of cultural psychology. New Ideas in Psychology, 28(1), 79-93.

21. Glaveanu, V.(2014). The psychology of creativity: a critical reading. Creativity: TheoriesResearch-Applications, 1(1), 10-32.

22. González, N. (2012). Alfabetización para una cultura social, digital, mediática y en red. Revista Española de Documentación Cientifica, número monográfico, 17-45.

23. Gutiérrez, A. y Tyner, K. (2012). Educación para los medios, alfabetización mediática y competencia digital. Comunicar, 38, 31-39.

24. Ipri, T. (2010). Introducing transliteracy. What does it mean to academic libraries? College $\mathcal{E}^{\circ}$ Research Libraries Nerws, 71(10), 532-567.

25. Lee, A. y So, C. (2014). Alfabetización mediática y alfabetización informacional: similitudes y diferencias. Comunicar, 42, 137-146.

26. López, L. y Aguaded, M. (2015). La docencia sobre alfabetización mediática en las facultades de Educación y Comunicación. Comunicar, 44, 187-195.

27. Moneréo, C. y Fuentes, M. (2008). La enseñanza y el aprendizaje de estrategias de búsqueda y selección de información en entornos virtuales. En C. Coll y C. Moneréo (eds.), Psicología de la educación virtual (pp. 386-408). Madrid: Morata.

28. Moneréo, C. y Badia, A. (2012). La competencia informacional desde una perspectiva psicoeducativa: enseñanza basada en la resolución de problemas prototípicos y emergentes. Revista Española de Documentación Cientifica, número monográfico, 75-99.
29. Pasadas, C. (2010). Multialfabetización y redes sociales en la universidad. Revista de Universidad y Sociedad del Conocimiento, 7(2). Recuperado de http://rusc.uoc.edu/index.php/rusc/article/ viewFile/v7n2-pasadas/v7n2-pasadas

30. Peña, J. et al. (2011) A review of the socio-cognitive and creative processes in the education mediated by digital environments. Tesis Psicológica, 6, 14-35.

31. Pérez-Mateo, M., Romero, M.y Romeu, T. (2014). La construcción colaborativa de proyectos como metodología para adquirir competencias digitales. Comunicar, 42, 15-24.

32. Sierra, C. (2011). La educación virtual como favorecedora del aprendizaje autónomo. Revista Panorama, 5(9), 75-87.

33. Steinbeck, R. (2011). El design thinking como estrategia de creatividad en la distancia. Comunicar, 37, 27-35.

34. Toro, J. M. (2004). Crea-ti-vida-d, cuerpo y comunicación. Comunicar, 23, 151-159.

35. Unesco (2009). Acerca del Programa Información para Todos. Recuperado de: http://www. unesco.org/new/es/communication-and-information/intergovernmental-programmes/ information-for-all-programme-ifap/priorities/ information-literacy/

36. Wilson, C. (2012). Alfabetización mediática e informacional: proyecciones didácticas. Comunicar, 39, 15-24.

.

